\title{
Toxicidade Cutânea dos Inibidores de Checkpoint Imunológico: Uma Revisão Narrativa
}

\section{Cutaneous Toxicity of Immune Checkpoint Inhibitors: A Narrative Review}

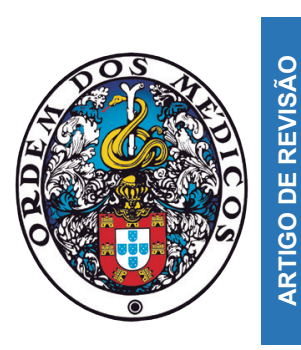

\author{
Nuno GOMES $\triangle^{1}$, Vincent SIBAUD ${ }^{2,3}$, Filomena AZEVEDO ${ }^{1}$, Sofia MAGINA ${ }^{1,4}$ \\ Acta Med Port 2020 May;33(5):335-343 - https://doi.org/10.20344/amp.12424
}

\begin{abstract}
RESUMO
Introdução: Os inibidores de checkpoint imunológico revolucionaram o tratamento anti-neoplásico. Nos últimos anos, a European Medicines Agency e a United States Food and Drug Administration aprovaram inibidores de vários checkpoint imunológicos, nomeadamente do antigénio 4 associado aos linfócitos T citotóxicos e da proteína 1 de morte celular programada ou o seu ligante. Apesar dos benefícios que acrescentam no tratamento de várias neoplasias, o bloqueio dos checkpoint imunológicos também se pode associar a múltiplos efeitos adversos imunorrelacionados.
\end{abstract}

Material e Métodos: Foi efetuada uma pesquisa da literatura da base de dados PubMed sobre a toxicidade cutânea dos inibidores de checkpoint imunológico até 30 de abril de 2019.

Resultados e Discussão: Foram triados 380 artigos em primeira análise, dos quais 75 constituem a base desta revisão bibliográfica. Os anticorpos monoclonais inibidores de checkpoint imunológico produzem os seus efeitos benéficos através da ativação do sistema imunológico. Desta ativação resultam também efeitos adversos que podem afetar qualquer órgão, sendo a toxicidade cutânea a mais frequente e precoce. Os efeitos adversos imunorrelacionados dos inibidores da proteína 1 de morte celular programada ou o seu ligante e inibidores do antigénio 4 associado aos linfócitos T citotóxicos são similares (efeito de classe), apesar da aparente maior toxicidade cutânea dos inibidores do antigénio 4 associado aos linfócitos T citotóxicos (ou do seu uso em combinação com os inibidores da proteína 1 de morte celular programada ou o seu ligante). Os efeitos adversos cutâneos mais comuns são o exantema maculopapular e o prurido, mas estão descritos outros mais característicos (reação liquenóide ou psoriasiforme, vitiligo e sarcoidose, entre outros) ou localizados às faneras e/ou mucosa oral, que estão aparentemente subestimados.

Conclusão: Dada a elevada frequência da toxicidade cutânea associada aos novos inibidores de checkpoint imunológico e respetivo impacto na qualidade de vida, o seu reconhecimento precoce e a abordagem adequada são cruciais no tratamento do doente oncológico. A observação pelo dermatologista deve ser providenciada em doentes com determinadas toxicidades.

Palavras-chave: Antineoplásicos/efeitos adversos; Doenças da Pele/induzidas quimicamente; Efeitos Colaterais e Reações Adversas Relacionados a Medicamentos; Imunomodelação/efeitos dos fármacos; Neoplasias/quimioterapia; Receptor de Morte Celular Programada 1 /antagonistas \& inibidores

\section{ABSTRACT}

Introduction: Immune checkpoint inhibitors revolutionized anti-neoplastic treatment. Recently, the European Medicines Agency and the United States Food and Drug Administration approved inhibitors of various immune checkpoints, namely the cytotoxic T-lymphocyte-associated protein 4, programmed cell death protein 1 and its ligand. Despite the added benefits in the treatment of several neoplasms, immune checkpoint blockade may also be associated with multiple immune-related adverse events.

Material and Methods: A literature review in PubMed database on the cutaneous toxicity of immune checkpoint inhibitors was performed until April 30, 2019.

Results and Discussion: A total of 380 articles were initially screened, of which 75 are the basis of this bibliographic review. The immune checkpoint inhibitors monoclonal antibodies produce their beneficial effects by activating the patient's immune system. This activation also results in adverse events that can affect any organ, whereas cutaneous toxicity is the most frequent and precocious. The adverse events of the programmed cell death protein 1 and its ligand and of the cytotoxic T-lymphocyte-associated protein 4 are similar (class effect), despite the apparent higher skin toxicity of inhibitors of the cytotoxic T-lymphocyte-associated protein 4 (or its use in combination with inhibitors of programmed cell death protein 1 and its ligand). The most common cutaneous toxicities are maculopapular exanthema and pruritus, but other more specific adverse effects (e.g. lichenoid or psoriasiform reaction, vitiligo, sarcoidosis, among others) or located in the oral mucosa and/or adnexa are underreported.

Conclusion: Given the high rate of cutaneous toxicity associated with new immune checkpoint inhibitors and their impact on quality of life, their early recognition and appropriate approach are crucial in the treatment of cancer patients. Observation by a dermatologist should be provided in patients with certain toxicities.

Keywords: Antineoplastic Agents/adverse effects; Immunomodulation/drug effects; Neoplasms/drug therapy; Programmed Cell Death 1/antagonists \& inhibitors; Receptor Skin Diseases/chemically induced

\section{INTRODUÇÃO}

Os anticorpos monoclonais que inibem determinados checkpoint imunológicos representam um tratamento recente e inovador em várias neoplasias. Devido ao seu me-

canismo de ação, os inibidores de checkpoint imunológico (ICI) causam múltiplos efeitos adversos imunorrelacionados (EAir), dos quais os cutâneos são os mais comuns e

1. Departamento de Dermatovenereologia. Centro Hospitalar Universitário de São João. Porto. Portugal.

2. Departamento de Dermatologia Oncológica. Instituto Claudius Regaud. Toulouse. França.

3. Departamento de Dermatologia Oncológica. Instituto Universitário de Cancro de Toulouse Oncopole. Toulouse. França.

4. Departamento de Dermatovenereologia. Faculdade de Medicina. Universidade do Porto. Porto. Portugal.

$\triangle$ Autor correspondente: Nuno Gomes. nunompretogomes@gmail.com

Recebido: 07 de junho de 2019 - Aceite: 10 de setembro de 2019 | Copyright @ Ordem dos Médicos 2020 
precoces. ${ }^{1}$ Assim sendo, o seu reconhecimento e abordagem adequada são cruciais no tratamento do doente oncológico. Ao longo do texto, os autores apresentam a principal toxicidade cutânea associada aos $\mathrm{ICl}$ e o melhor modo de abordagem.

A classificação por graus da toxicidade cutânea, patente ao longo do texto e na Tabela 1 , baseou-se na classificação do National Cancer Institute - Common Termino- logy Criteria for Adverse Events, versão 4. O texto inclui várias referências de artigos que não foram redigidos por dermatologistas e que aplicavam indiscriminadamente o termo 'rash' para descrever diferentes padrões de reação cutânea. Os autores aplicaram o termo 'exantema' em sua substituição ou outros termos mais específicos do léxico dermatológico. As toxicidades cutâneas descritas ao longo do texto são apresentadas na Tabela 2.

Tabela 1 - Abordagem das duas principais toxicidades cutâneas dos ICl (adaptado de Puzanov I et al e Sibaud $V$ et a ${ }^{12}$ )

\begin{tabular}{|c|c|c|c|}
\hline \multicolumn{4}{|c|}{ Exantema maculopapular } \\
\hline Grau & Descrição & Abordagem & Referenciar a dermatologista? \\
\hline 1 & $\begin{array}{l}\text { Máculas/pápulas que afetam } \\
<10 \% \text { da área de superfície } \\
\text { corporal [ASC] com ou sem } \\
\text { sintomas (prurido, queimor) }\end{array}$ & $\begin{array}{l}\text { - } \quad \text { Continuar ICl; } \\
\text { - Antihistamínicos [AH] orais (cetirizina/loratadina } \\
10 \text { mg diário; hidroxizina } 10-25 \text { mg diário); } \\
\text { - } \text { Glicocorticóides [GC] tópicos } \\
\text { (corpo - classe I - creme ou pomada de } \\
\text { propionato clobetasol, propionato halobetasol } \\
\text { ou dipropionato betametasona; face - classe } \\
\text { V/VI - creme de aclometasona, desonido ou } \\
\text { hidrocortisona 2,5\%); } \\
\text { - Reavaliar após } 2 \text { semanas e monitorizar. }\end{array}$ & Não \\
\hline 2 & $\begin{array}{l}\text { Máculas/pápulas que afetam } \\
10 \%-30 \% \text { ASC com ou sem } \\
\text { sintomas (prurido, queimor); } \\
\text { afeta a capacidade funcional }\end{array}$ & $\begin{array}{l}\text { - Continuar ICl, reavaliar em 1-2 semanas e } \\
\text { monitorizar; } \\
\text { - } \quad \text { Referenciação não urgente à Dermatologia; } \\
\text { - } \mathrm{AH} \text { orais e GC tópicos = Grau } 1\end{array}$ & Sim \\
\hline 3 & $\begin{array}{l}\text { Máculas/pápulas que afetam } \\
\geq 30 \% \text { ASC com ou sem } \\
\text { sintomas (prurido, queimor); } \\
\text { afeta o auto-cuidado }\end{array}$ & $\begin{array}{ll}\text { - } & \text { Descontinuar ICl; } \\
\text { - } & \text { Referenciação urgente à Dermatologia; } \\
\text { - } & \text { Excluir hipersensibilidade sistémica; } \\
\text { - } & \text { AH orais = Grau } 1 ; \\
& \text { Prednisolona } 0,5-1,0 \mathrm{mg} / \mathrm{kg} / \text { dia (ou dose } \\
& \text { equivalente de metilprednisolona) até o exantema } \\
& \text { melhorar para } \leq 1 ; \\
\text { - } & \text { Retomar o ICl após } 1 \text { mês de desmame de } \\
& \text { GC e dose total prednisolona }<10 \mathrm{mg} \text {; Se } \\
& \text { persistência ou agravamento - descontinuar } \\
& \text { permanentemente } \mathrm{ICl} .\end{array}$ & Sim \\
\hline \multicolumn{4}{|c|}{ Prurido } \\
\hline 1 & $\begin{array}{l}\text { Leve ou localizado; } \\
\text { intervenção tópica indicada }\end{array}$ & $\begin{array}{l}\text { Emolientes em creme ou pomada, produtos sem } \\
\text { fragrância; } \\
\text { GC tópicos (corpo - classe I - creme ou pomada } \\
\text { de propionato clobetasol, propionato halobetasol } \\
\text { ou dipropionato betametasona; face - classe } \\
\mathrm{V} / \mathrm{VI} \text { - creme de aclometasona, desonido ou } \\
\text { hidrocortisona } 2,5 \% \text { ) } \mathrm{AH} \text { orais (cetirizina/ } \\
\text { loratadina } 10 \mathrm{mg} \text { diário; hidroxizina } 10 \text { - } 25 \mathrm{mg} \\
\text { diário). }\end{array}$ & Não \\
\hline 2 & $\begin{array}{l}\text { Intenso ou disseminado; } \\
\text { intermitente; alterações } \\
\text { cutâneas por coceira (edema, } \\
\text { pápulas, escoriações, } \\
\text { liquenificação, crostas); } \\
\text { intervenção oral indicada; } \\
\text { afeta a capacidade funcional }\end{array}$ & $\begin{array}{l}\text { - Referenciação não urgente à Dermatologia; } \\
\text { - } \quad \text { AH orais e GC tópicos = Grau } 1 \\
\text { equivalente de metilprednisolona) desmame em } \\
\text { equal } \\
2 \text { semanas. }\end{array}$ & Sim \\
\hline 3 & $\begin{array}{l}\text { Intenso ou disseminado; } \\
\text { constante; afeta o auto- } \\
\text { cuidado ou o sono; GC oral } \\
\text { ou terapia imunossupressora } \\
\text { indicada }\end{array}$ & $\begin{array}{l}\text { - Referenciação urgente à Dermatologia; } \\
\text { - Agonista GABA (pregabalina, gabapentina 100- } \\
200 \mathrm{mg} \text { TID); } \\
\text { GC oral - prednisolona } 0,5-1,0 \mathrm{mg} / \mathrm{kg} / \mathrm{dia} \text { (ou } \\
\text { equivalente de metilprednisolona) desmame em } \\
2 \text { semanas. }\end{array}$ & Sim \\
\hline
\end{tabular}


Tabela 2 - Toxicidades cutâneas específicas dos ICl

\begin{tabular}{ll} 
Exantema cutâneo & Exantema maculopapular \\
& Reação liquenóide \\
& Reação psoriasiforme \\
Prurido & Toxicidade cutânea com atingimento sistémico \\
Vitiligo & \\
Dermatoses auto-imunes & Penfigóide bolhoso \\
& Dermatomiosite \\
& Lúpus eritematoso cutâneo \\
& Síndrome de Sjögren \\
& Vasculite \\
Outras toxicidades cutâneas & Doença de Grover \\
& Sarcoidose \\
& Dermatoses neutrofílicas \\
& Toxicidade do cabelo e unhas \\
& Toxicidade da mucosa oral \\
\hline
\end{tabular}

\section{MATERIAL E MÉTODOS}

Foi efetuada uma pesquisa da literatura para identificar artigos científicos sobre a toxicidade cutânea dos inibidores de checkpoint imunológico. Os autores identificaram resumos e textos completos da base de dados PubMed até 30 de abril de 2019. A pesquisa foi restrita para artigos escritos em Inglês e Português. Os termos MeSH usados na pesquisa incluíram: 'Cytotoxic T-lymphocyte-associated protein 4 Antigen/antagonists \& inhibitors', 'Dermatologic Agents/ therapeutic use', 'Immunotherapy/adverse effects', 'Programmed Cell Death 1 Receptor/antagonists \& inhibitors' e 'Treatment Outcome'. Adicionalmente, as listas de referências dos estudos identificados foram revistas manualmente para identificar publicações complementares.

Foram triados 380 artigos em primeira análise. Os artigos incluídos nesta revisão respeitavam os seguintes critérios: [1] meta-análises, editoriais e revisões de peritos; [2] desenho observacional (prospetivo, retrospetivo, série de casos ou relato de caso); [3] intervencional. A extração de dados foi efetuada independentemente por dois investigadores (NG e SM), com discrepâncias resolvidas pelo autor sénior (SM).

\section{RESULTADOS E DISCUSSÃO}

\section{Caraterização e aplicações terapêuticas dos ICI}

Os $\mathrm{ICl}$ são fármacos relativamente recentes e a caraterização dos seus efeitos adversos (EA) está ainda em expansão. Os checkpoint imunológicos são moléculas da superfície das células do sistema imunológico, que enviam estímulos co-inibitórios e atenuam a resposta imune. ${ }^{2}$ São essenciais na homeostasia do sistema imunológico, pois infra-regulam a ativação e proliferação de células $T$, permitindo que este seja tolerante aos auto-antigénios mas também a determinados tipos de células tumorais. Através da ativação das células T CD4+/CD8+ citotóxicas, os ICl alteram a ação do sistema imunológico e desencadeiam a atividade anti-tumoral. ${ }^{3} \mathrm{O}$ melanoma maligno é o protótipo de neoplasia com propriedades imunogénicas e a imunoterapia foi inicialmente desenvolvida neste contexto, com o uso da interleucina-2. ${ }^{2}$

Os $\mathrm{ICl}$ são anticorpos monoclonais que têm como principais alvos o antigénio 4 associado aos linfócitos T citotóxicos (CTLA-4) e a proteína 1 de morte celular programada (PD-1) ou o seu ligante (PD-L1). ${ }^{4}$ Estes fármacos revolucionaram o tratamento de diversas neoplasias, nomeadamente o melanoma metastático, cancro de pulmão não pequenas células e carcinoma urotelial. Atualmente, é consensual que os ICI (IPD1, IPDL1 e ICTLA4) são a abordagem terapêutica sistémica mais promissora para alcançar respostas duradouras ou até cura de muitos tipos de neoplasias. ${ }^{5,6}$

A United States Food and Drug Administration (US FDA) e a European Medicines Agency (EMA) aprovaram seis ICI para o tratamento de diversas neoplasias desde 2011. O ipilimumab (ICTLA4) foi inicialmente aprovado no melanoma, a que se seguiram o nivolumab e pembrolizumab (IPD1) no melanoma, cancro pulmão não pequenas células, linfoma de Hodgkin, carcinoma urotelial e carcinoma espinocelular da cabeça e pescoço. Posteriormente, a associação de ipilimumab e nivolumab foi também aprovada. Por último, o atezolizumab, avelumab e durvalumab (IPDL1) receberam aprovação no cancro pulmão não pequenas células, carcinoma urotelial e/ou carcinoma de células de Merkel. ${ }^{7}$ Prevê-se que, nos anos vindouros, quer estes quer outros inibidores de checkpoint imunológico serão aprovados para o tratamento destas e outras neoplasias.

\section{Efeitos adversos imunorrelacionados dos $\mathrm{ICI}$}

Dado terem um mecanismo de ação único, os $\mathrm{ICl}$ têm um perfil de segurança muito específico. Assim, desencadeiam EA de natureza imunológica, mediados pela ativação das células T CD4+/CD8+ citotóxicas que têm como alvo 
auto-antigénios presentes em tecidos saudáveis (EAir). ${ }^{3,7}$ Podem envolver qualquer sistema orgânico, mas as toxicidades cutânea, gastrointestinal, hepática e endocrinológica predominam. No tratamento do melanoma, os EA cutâneos podem surgir nas primeiras semanas de terapia, mas também podem ter início após a descontinuação do tratamento. A toxicidade cutânea dos $\mathrm{ICl}$ é mais frequente nos doentes com melanoma do que em outras neoplasias. ${ }^{6}$

Os EA causados pelos ICl têm um início tipicamente mais tardio e duração prolongada comparativamente aos EA associados à quimioterapia. A abordagem eficaz destes EA assenta no seu reconhecimento precoce e intervenção rápida com imunossupressão e/ou estratégias de imunomodulação. Apesar da maioria dos EAir ser leve a moderado, podem ocorrer EAir potencialmente fatais, como a necrólise epidérmica tóxica (NET), e a morte associada aos ICI foi reportada em até $2 \%$ dos doentes em ensaios clínicos. ${ }^{1}$ Apesar de pertencerem à mesma classe de ICI, os ICTLA4, IPD1 e IPDL1 têm diferentes perfis de EAir. Em geral, os IPD1 têm menos EA do que o ipilimumab (EA graus 3 e 4 de $10 \%-15 \%$ versus $20 \%$ - $30 \%$ respetivamente) e certamente menos do que a combinação ipilimumab/nivolumab (EA graus 3 e 4 de $55 \%$ ). ${ }^{8}$

\section{Toxicidades cutâneas específicas dos ICI}

Os EA cutâneos afetam até metade dos doentes sob $\mathrm{ICl}$, independentemente da neoplasia em tratamento, correspondendo a um efeito de classe. A toxicidade cutânea (de qualquer grau) é observada em $30 \%$ - 40\% de doentes sob IPD1/IPDL1 e em cerca de $50 \%$ de doentes sob ipilimumab. ${ }^{1}$ Em geral, o bloqueio da CTLA-4 provoca reações similares ao bloqueio do PD-1; porém, os IPD1 são geralmente melhor tolerados do que os ICTLA4. Ambas as reações tendem a ser tardias, com os ICTLA4 a causar exantema após cerca de um mês de fármaco e os IPD1 um pouco mais tarde. ${ }^{9,10}$ Apesar da frequência com que ocorrem, a maioria das toxicidades é autolimitada e os ICI apresentam um perfil de toxicidade cutânea aceitável. . $^{3,8} \mathrm{O}$ exantema e o prurido lideram os cinco principais EAir relatados nos ensaios clínicos para esta classe terapêutica. ${ }^{11,12}$ O dermatologista pode desempenhar um papel importante na abordagem das toxicidades cutâneas, seja a nível diagnóstico (pesquisa de anticorpos específicos em dermatoses autoimunes, realização de biópsia cutânea, etc.), seja a nível terapêutico (tratamento imunomodulador ou imunossupressor tópico e/ou sistémico).

Desde há alguns anos, as terapêuticas de combinação têm sido testadas em ensaios clínicos no tratamento do melanoma metastático. Uma revisão de 1551 doentes com melanoma avançado concluiu que os EAir ocorreram mais frequentemente com a combinação de nivolumab e ipilimumab, tendo o seu início sido mais rápido e o seu curso habitualmente mais grave. As toxicidades cutâneas afetaram $62 \%$ dos doentes em terapia combinada, tendo sido os EA mais comuns. ${ }^{13}$ Apesar de terem algumas reações que Ihes são específicas, esta classe de fármacos pode causar toxidermias que ocorrem tipicamente com outras classes.
O tratamento de cada toxicidade deve ser individualizado; a Tabela 1 discrimina a abordagem das toxicidades cutâneas mais frequentes. Por último, salienta-se que vários EAir podem ocorrer simultaneamente no mesmo doente, surgindo concomitante ou sequencialmente, e que nem sempre é possível catalogar com precisão os vários tipos de EA.

\section{Exantema cutâneo}

\subsection{Exantema maculopapular}

O exantema maculopapular representa a toxicidade cutânea mais comum associada aos ICl.3,14,15 Os ICTLA4 são os mais associados a exantema maculopapular, secundados pelos IPD1; o risco parece ser menor com os IPDL1. ${ }^{12,16} \mathrm{~A}$ apresentação clínica é habitualmente inespecífica, caraterizada por exantema morbiliforme que habitualmente se inicia no tronco e se dissemina para as extremidades, sendo assintomático ou ocasionalmente pruriginoso. ${ }^{9,11,17,18} \mathrm{~A}$ face é habitualmente poupada. O exantema pode piorar após cada ciclo de tratamento e alguns autores reportaram lesões sobretudo nas áreas fotoexpostas. ${ }^{19,20}$

A biópsia cutânea revela uma dermatite linfocítica de interface, perivascular e perianexial, com poucos plasmócitos e eosinófilos. Deve ser efetuada na presença de lesões atípicas, exantema grau 2 ou 3 persistente e toxicidade cutânea com atingimento sistémico. ${ }^{12,21} \mathrm{Em}$ todos os casos, o exantema maculopapular inespecífico pode representar a manifestação inicial de uma dermatose mais específica induzida por $\mathrm{ICl}$, incluindo reação liquenóide, psoríase (de novo ou agravada), doença de Grover, penfigóide bolhoso ou toxidermias potencialmente fatais. ${ }^{3}$

\subsection{Reação liquenóide}

As reações liquenóides aos $\mathrm{ICl}$ são particularmente observadas com IPD1/IPDL1. O diagnóstico é geralmente efetuado após análise histológica e estima-se que a sua incidência esteja subestimada.,12 As reações liquenóides associadas aos IPD1 localizam-se habitualmente no tronco e extremidades e apresentam-se como múltiplas pápulas e placas discretas, eritematosas ou por vezes violáceas. A face e membranas mucosas estão habitualmente poupadas, ${ }^{22,23}$ apesar de lesões liquenóides da mucosa oral e/ou anogenital terem sido recentemente descritas. ${ }^{24,25}$

O início da reação liquenóide é habitualmente mais tardio do que a do exantema maculopapular inespecífico e a sua incidência aumenta a uma taxa aproximadamente constante após iniciar o tratamento. É essencial rever a medicação completa dos doentes, pois muitas reações liquenóides podem ser induzidas por fármacos que não os $\mathrm{ICl}$. Histologicamente, apresenta-se como dermatite liquenóide de interface com infiltrado de linfócitos $\mathrm{T}$ em banda na derme superior, podendo ocorrer degeneração vacuolar com disrupção parcial da camada basal. ${ }^{12,17,21,26}$ A reação liquenóide é a alteração histológica mais comum em doentes sob IPD1. ${ }^{24,25}$

\subsection{Reação psoriasiforme}

A reação psoriasiforme é uma toxicidade cutânea bem 
estabelecida dos IPD1 e ICTLA4, e os achados histológicos são muito similares aos classicamente observados na psoríase. ${ }^{3} \mathrm{~A}$ dermatite psoriasiforme pode ser causada por $\mathrm{ICI}$ no contexto de várias neoplasias e manifestar-se como psoríase inaugural ou como agravamento de uma psoríase prévia, sendo esta a forma mais comum. ${ }^{27}$ Inicia-se habitualmente no segundo mês de tratamento, mas pode surgir mais tarde, caso seja inaugural. Tem especial predileção pelo couro cabeludo e a forma clínica mais comum é a psoríase em placas. ${ }^{11,28} \mathrm{~A}$ patogénese da reação psoriasiforme permanece especulativa. Porém, foi claramente demonstrado que a psoríase se associa fortemente aos linfócitos Th17 e produção de interleucinas (IL) [IL-17 e IL-22], que são, pelo menos em parte, infra-regulados pela via PD-1. A inibição dessa via pelos $\mathrm{ICl}$ aumenta a ativação de células Th17, induzindo as lesões de psoríase. ${ }^{27,29}$

\subsection{Toxicidade cutânea com atingimento sistémico}

A apresentação inicial de uma toxicidade cutânea potencialmente fatal, como a síndrome Stevens-Johnson/ necrólise epidérmica tóxica (SSJ/NET), pode ser sob a forma de um exantema maculopapular que progride com a formação de vesículas e/ou bolhas associadas a dor cutânea. ${ }^{14} \mathrm{O}$ exantema cutâneo sob a forma de SSJ/NET foi raramente reportado associado aos $\mathrm{ICI}$, seja com ICTLA4, ${ }^{30}$ seja com IPD1, ${ }^{31-34}$ tendo alguns casos sido fatais. O tempo de aparecimento do exantema foi muito variável, mas foram descritos casos com início muito tardio. ${ }^{33} \mathrm{O}$ tratamento incluiu corticoterapia oral e/ou intravenosa, bem como ciclosporina, ${ }^{32}$ infliximab ${ }^{34}$ ou imunoglobulina intravenosa. ${ }^{34}$ Casos de reação farmacológica com eosinofilia e sintomas sistémicos (DRESS) associados ao nivolumab e/ou ipilimumab também foram reportados, ${ }^{35}$ bem como raros casos de pustulose generalizada exantemática aguda (PGEA). ${ }^{36,37}$

Vivar et al reportaram um caso fatal onde se comprovou existir na biópsia cutânea um aumento do número de linfócitos CD8+ na junção dermo-epidérmica e da expressão do PD-L1 em linfócitos e em queratinócitos. ${ }^{34}$ Tais achados foram corroborados por Goldinger SM et al, que reportaram que o perfil histológico e a análise genética das biópsias cutâneas de doentes com exantema maculopapular é comum às reações tipo-necrólise epidérmica tóxica, pelo que se presume que a interação PD-1/PD-L1 possa ser essencial para preservar a integridade epidérmica durante as reações cutâneas inflamatórias. ${ }^{31} \mathrm{Na}$ presença de um exantema deste tipo, o $\mathrm{ICl}$ deve ser descontinuado permanentemente. ${ }^{38,39}$

\section{Prurido}

O prurido é referido por até $35 \%$ dos doentes sob ipilimumab, 20\% sob IPD1 e 33\% com a combinação de ambos $^{8,12}$; o risco é um pouco menor em doentes sob IPDL1. ${ }^{40}$ O prurido associa-se habitualmente ao exantema maculopapular, apesar de o poder preceder ou ocorrer sem dermatose. O couro cabeludo é frequentemente envolvido e a face é geralmente poupada. ${ }^{3}$

O prurido leve a moderado deve ser tratado sintomati- camente com glicocorticóides tópicos e/ou antihistamínicos orais. Em casos resistentes, a gabapentina, o aprepitant e a doxepina podem ter efeitos benéficos, bem como a fototerapia. ${ }^{26,41} \mathrm{O}$ prurido de grau 3 (Tabela 1 ) pode obrigar à interrupção do $\mathrm{ICl}$ até que este EA seja reduzido para grau menor ou igual a 1. Por último, deve-se ressalvar que o prurido causado por IPD1/IPDL1 pode representar a forma inicial não bolhosa de penfigóide bolhoso (PB). De facto, os autoanticorpos contra BP180 podem estar presentes previamente ao desenvolvimento de bolhas. Assim, doentes com prurido intermitente ou constante devem ser avaliados por dermatologista. ${ }^{42}$

\section{Vitiligo}

O vitiligo surge mais comummente associado aos IPD1 do que aos ICTLA4. ${ }^{12,14,43}$ Apesar do vitiligo associado aos $\mathrm{ICl}$ ter sido descrito quase exclusivamente em doentes com melanoma, existem relatos recentes deste EAir em outras neoplasias. ${ }^{44,45}$ Uma vez que a maioria dos estudos com IPDL1 não foram realizados no tratamento do melanoma, é expectável que os casos de vitiligo em doentes sob este ICI ainda sejam escassos. ${ }^{3} \mathrm{O}$ vitiligo associado aos $\mathrm{ICl}$ pode corresponder a uma reação cruzada contra os antigénios que são partilhados pelas células do melanoma e os melanócitos normais, como sejam o Melan-A, gp100 e proteínas associadas à tirosinase 1 e $2 .^{14,46}$

O vitiligo pode ser assintomático ou cursar com queixas de prurido precedente; a apresentação é mais comummente bilateral. ${ }^{3} \mathrm{O}$ tempo de aparecimento é variável mas pensa-se que a incidência aumente a uma taxa aproximadamente constante após o início do ICl, surgindo habitualmente a partir do segundo mês de tratamento, com $25 \%$ dos doentes a desenvolver este EA em 10 meses após início do fármaco. ${ }^{24,47}$ Larbasal et al constataram que as lesões de vitiligo associado aos $\mathrm{ICI}$ eram clinicamente (mais localizadas e menos simétricas, predomínio nas áreas foto-expostas, ausência de fenómeno de Koebner) e histologicamente (sobre-expressão do receptor da quimiocina 3 (CXCR3) pelo infiltrado de células T CD8+) diferentes do vitiligo clássico, o que pode indiciar um mecanismo fisiopatológico distinto. ${ }^{48,49}$ Pensa-se que é um EAir subestimado pois pode facilmente não ser referido ou observado, exceto se for efetuado um exame objetivo completo. ${ }^{12}$ As lesões frequentemente persistem após a interrupção do tratamento. ${ }^{14}$

\section{Dermatoses auto-imunes \\ 4.1 Penfigóide bolhoso}

As dermatoses bolhosas auto-imunes são EAir raros do tratamento com $\mathrm{ICl}$, mas cuja referenciação à Dermatologia é essencial. Nos últimos anos, surgiram vários relatos de PB sob pembrolizumab e nivolumab. ${ }^{50,51} \mathrm{O}$ PB associado a IPD1/IPDL1 pode persistir durante vários meses após descontinuação do fármaco, não havendo caraterísticas específicas que diferenciem o PB clássico do PB induzido por estes fármacos. ${ }^{42}$ Inicialmente, considerava-se que o PB era um efeito específico dessa subclasse de ICI. 
Porém, recentemente surgiram relatos de doentes sob ipilimumab que desenvolveram PB..$^{52}$ Clinicamente, a maioria dos relatos descreve doentes com lesões pruriginosas, nem sempre acompanhadas por bolhas mas habitualmente com bolhas que surgem 6 - 8 meses após início do tratamento, habitualmente sem afetar as mucosas. ${ }^{42,50-52}$

Alguns autores adiantaram que o PB associado ao bloqueio da via $\mathrm{PD}-1 / \mathrm{PD}-\mathrm{L} 1$ pode ser mediado pelas células $\mathrm{T}$, que passam a ter como alvo a proteína BP180 das células tumorais e da membrana basal da epiderme. ${ }^{42}$ Apesar desta evidência, o mecanismo subjacente ao PB induzido por $\mathrm{ICl}$ ainda não foi completamente caraterizado. ${ }^{3}$

\subsection{Dermatomiosite}

A apresentação cutânea clássica de dermatomiosite foi esporadicamente associada ao tratamento com ICl. ${ }^{3}$ As lesões podem aparecer logo no primeiro mês do fármaco ${ }^{53} \mathrm{ou}$ alguns meses mais tarde ${ }^{54}$, tendo sido descritas quer com IPD1 quer com ICTLA4. A maioria dos casos correspondeu a dermatomiosite cutânea com miosite associada e levou à descontinuação do fármaco implicado e tratamento sintomático com corticoterapia oral ou endovenosa.

\subsection{Lúpus eritematoso cutâneo}

Nos últimos anos, também o lúpus eritematoso cutâneo, habitualmente na forma subaguda, foi descrito associado ao pembrolizumab ${ }^{55}$ e nivolumab. ${ }^{56}$ Este EAir ocorreu nos primeiros meses de tratamento ou após a sua suspensão. A maioria dos doentes não tinha antecedentes de patologia autoimune e desenvolveu lúpus eritematoso cutâneo com anticorpos ANA positivos, sem envolvimento sistémico. Tal como referido anteriormente, a interação PD-1/PD-L1 regula a indução e manutenção da tolerância periférica e protege os tecidos do ataque autoimune, pelo que o bloqueio dessa via pode resultar neste tipo de patologia. ${ }^{57}$

\subsection{Síndrome de Sjögren}

A síndrome de Sjögren, com várias apresentações clínicas, é um EAir raro dos ICl. Num estudo retrospetivo francês, foram descritos dois casos que se apresentaram com xerostomia e xeroftalmia e anticorpos antinucleares (ANA) e anti-antigénio $A$ relacionado com a síndrome de Sjögren (anti-SSA) positivos; ambos obrigaram à interrupção do tratamento. ${ }^{58} \mathrm{Um}$ estudo americano relatou quatro doentes com síndrome sicca e hipofunção salivar grave, confirmados por sialografia e ecografia, também com anticorpos antinucleares positivos. ${ }^{59}$ Este tipo de EAir é provavelmente subestimado. Admite-se que a patogénese autoimune destes EA difira das manifestações da doença reumática clássica, ${ }^{60}$ tendo sido demonstrado que o PD-1 é expresso nos linfócitos T e o PD-L1 nas células epiteliais das glândulas salivares inflamadas de doentes com síndrome de Sjögren. Este facto implica que a alteração da função da via PD-1/ PD-L1 possa estar relacionada com a falência na repressão de linfócitos. ${ }^{61}$

\subsection{Vasculite}

A vasculite de pequenos vasos foi observada quer com IPD1, sob a forma de livedo ou necrose digital, ${ }^{3}$ quer com ICTLA4, com posterior progressão para necrólise epidérmica tóxica. ${ }^{30}$ Recentemente, uma revisão bibliográfica reportou um total de 53 casos de vasculite associados aos ICl. Apesar de a vasculite de grandes vasos e a vasculite do sistema nervoso central e periférico terem sido as formas mais frequentes, foram descritas vasculites de pequenos vasos com atingimento cutâneo. ${ }^{62}$

\section{Outras toxicidades cutâneas \\ 5.1 Doença de Grover}

Algumas doenças dermatológicas foram raramente associadas aos $\mathrm{ICl}$, amplificando o espetro de toxicidades possíveis destes fármacos. É o caso da doença de Grover ou dermatose acantolítica transitória, que foi especialmente associada ao ipilimumab ${ }^{63,64}$ ou ao pembrolizumab ${ }^{65}$; a apresentação clínica consistiu numa erupção vesiculosa ou papuloescamosa pruriginosa do tronco que ocorreu no segundo mês do fármaco ${ }^{63}$ e durou vários meses após a sua descontinuação ${ }^{64}$. O diagnóstico da doença de Grover exige biópsia cutânea e a sua incidência está provavelmente subestimada. $^{3}$

\subsection{Sarcoidose}

À semelhança da psoríase, a sarcoidose pode ocorrer como exacerbação de sarcoidose prévia ou aparecimento de novo da dermatose..$^{3,12}$ Os órgãos mais comummente afetados são os pulmões, o que implica o diagnóstico diferencial com metástases de melanoma em doentes sob ICI por esta neoplasia. ${ }^{66}$ No entanto, as lesões cutâneas também podem ser a forma de apresentação, frequentemente com nódulos eritematosos subcutâneos, com granulomas epitelióides não caseosos à histologia. ${ }^{67} \mathrm{~A}$ sarcoidose também se pode apresentar como síndrome de Löfgren ${ }^{3}$ ou sob a forma de pápulas, placas e lesões localizadas em cicatrizes ou tatuagens. ${ }^{68} \mathrm{~A}$ corticoterapia sistémica é a principal arma terapêutica e raramente este EA obriga à descontinuação do ICl. Alguns autores consideram a sarcoidose um EAir paradoxal, tendo em conta a supra-regulação de PD-1 pelos linfócitos TCD4+ em doentes com sarcoidose. ${ }^{69}$

\subsection{Dermatoses neutrofílicas}

Quer a síndrome de Sweet, quer o pioderma gangrenoso foram relatados em doentes sob $\mathrm{ICl} .{ }^{3,70} \mathrm{~A}$ síndrome de Sweet e a sua variante dermatose neutrofílica das mãos foram reportadas sobretudo com ipilimumab. ${ }^{71} \mathrm{O}$ pioderma gangrenoso também foi associado a esse $\mathrm{ICl}^{72}$ Os casos descritos responderam à corticoterapia oral. Dada a possível associação paraneoplásica de ambas as síndromes, é essencial estabelecer a etiologia das mesmas.

\subsection{Toxicidade do cabelo e unhas}

A alopecia foi descrita desde os primeiros ensaios clínicos com $\mathrm{ICl}$ e afeta até $2 \%$ dos doentes tratados com IPD1. ${ }^{17,18}$ Mais comummente, apresenta-se como alopecia 
areata focal ou universal, que surge a partir dos primeiros três meses de tratamento, é reversível após a descontinuação do mesmo e cursa com poliose (branqueamento localizado) aquando do recrescimento do cabelo. A fisiopatologia desta alopecia pode ser distinta da alopecia areata clássica, pois os $\mathrm{ICl}$ levam à ativação de respostas inflamatórias que expõem os antigénios do folículo piloso, para além de criarem um desequilíbrio entre o sistema imune e a auto-tolerância em indivíduos susceptíveis. Histologicamente, os achados incluem alopecia não cicatricial com infiltrado de células $\mathrm{T}$ perifolicular. ${ }^{73}$ Outro EA associado aos $\mathrm{ICl}$ é a repigmentação do cabelo: Rivera et al relataram recentemente 14 doentes sob IPD1/IPDL1 com repigmentação progressiva difusa do cabelo, com início nas regiões occipital e temporal. ${ }^{74}$

A toxicidade ungueal foi raramente reportada, podendo ser subvalorizada. Foram descritas alterações ungueais como onicosquizia (destacamento do bordo livre da unha) e múltiplos casos de distrofia ungueal, por vezes com onicomadese (descolamento da unha), onicólise difusa e paroníquia. ${ }^{3}$ A toxicidade ungueal associa-se mais frequentemente a outros fármacos quimioterápicos ou terapêuticas alvo.

\subsection{Toxicidade da mucosa oral}

Para além da reação liquenóide da mucosa oral ou da xerostomia no contexto da síndrome de Sjögren, previamente mencionadas, outras toxicidades da mucosa oral foram associadas a ICI. Quer os IPD1 quer os IPDL1 foram associados a estomatite inespecífica ou inflamação da mucosa oral em casos esporádicos. ${ }^{75} \mathrm{~A}$ xerostomia ligeira foi reportada em cerca de $6 \%$ de doentes sob nivolumab e até $7,2 \%$ sob pembrolizumab, ${ }^{75}$ não se associando às alterações imunológicas descritas na síndrome de Sjögren. A disgeusia afetou uma percentagem inferior ( 3\%) de doentes sob IPD1. ${ }^{3}$ Tanto a disgeusia como a xerostomia são menos frequentes sob ICTLA4. ${ }^{75}$

\section{REFERÊNCIAS}

1. Puzanov I, Diab A, Abdallah K, Bingham C, Brogdon C, Dadu R, et al. Managing toxicities associated with immune checkpoint inhibitors: consensus recommendations from the Society for Immunotherapy of Cancer (SITC) Toxicity Management Working Group. J Immunother Cancer. 2017;5:95.

2. Naidoo J, Page D, Wolchok J. Immune checkpoint blockade. Hematol Oncol Clin North Am. 2014;28:585-600.

3. Sibaud V. Dermatologic reactions to immune checkpoint inhibitors: skin toxicities and immunotherapy. Am J Clin Dermatol. 2018;19:345-61.

4. Mamalis A, Garcha M, Jagdeo J. Targeting the PD-1 pathway: a promising future for the treatment of melanoma. Arch Dermatol Res. 2014;306:511-9.

5. Blank C, Enk A. Therapeutic use of anti-CTLA-4 antibodies. Int Immunol. 2015;27:3-10.

6. Spain L, Diem S, Larkin J. Management of toxicities of immune checkpoint inhibitors. Cancer Treat Rev. 2016;44:51-60.

7. Hargadon K, Johnson C, Williams C. Immune checkpoint blockade therapy for cancer: an overview of FDA-approved immune checkpoint inhibitors. Int Immunopharmacol. 2018;62:29-39.

8. Haanen J, Carbonnel F, Robert C, Kerr K, Peters S, Larkin J, et al.

\section{CONCLUSÃO}

À medida que os ICl se vão estabelecendo como terapêuticas eficazes em vários tipos de neoplasias, aumenta o reconhecimento das múltiplas toxicidades cutâneas que lhes podem estar associadas e cresce o conceito de dermato-imuno-oncologia. Os EAir dos IPD1/IPDL1 e ICTLA4 são similares (efeitos de classe), apesar da aparente maior toxicidade cutânea dos ICTLA4 ou do seu uso em combinação com os IPD1/IPDL1. Os EAir mais comuns são o exantema maculopapular e o prurido, mas estão descritos outros EAir mais característicos (reação liquenóide, reação psoriasiforme, vitiligo, sarcoidose, entre outros) ou EAir localizados às faneras e/ou mucosa oral, que estão aparentemente subestimados. O reconhecimento precoce e abordagem adequada da toxicidade cutânea associada aos ICl são essenciais para prevenir o impacto negativo na qualidade de vida e no curso do tratamento da neoplasia. A integração de dermatologistas na abordagem multidisciplinar de doentes com neoplasias é essencial no diagnóstico e tratamento da toxicidade cutânea observada com os ICl.

\section{PROTEÇÃO DE PESSOAS E ANIMAIS}

Os autores declaram que os procedimentos seguidos estavam de acordo com os regulamentos estabelecidos pelos responsáveis da Comissão de Investigação Clínica e Ética e de acordo com a Declaração de Helsínquia da Associação Médica Mundial.

\section{CONFIDENCIALIDADE DOS DADOS}

Os autores declaram ter seguido os protocolos do seu centro de trabalho acerca da publicação de dados.

\section{CONFLITOS DE INTERESSE}

Os autores declaram não ter conflitos de interesses relacionados com o presente trabalho.

\section{FONTES DE FINANCIAMENTO}

Este trabalho não recebeu qualquer tipo de suporte financeiro de nenhuma entidade no domínio público ou privado.

Management of toxicities from immunotherapy: ESMO Clinical Practice Guidelines for diagnosis, treatment and follow-up. Ann Oncol. 2017;28:iv119-42.

9. Patel AB, Pacha O. Skin reactions to immune checkpoint inhibitors. Adv Exp Med Biol. 2018;995:117-29.

10. Robert C, Schachter J, Long G, Arance A, Grob J, Mortier L, et al. Pembrolizumab versus ipilimumab in advanced melanoma. N Engl J Med. 2015;372:2521-32

11. Patel A, Pacha O. Skin reactions to immune checkpoint inhibitors. Adv Exp Med Biol. 2017;995:175-84.

12. Sibaud V, Meyer N, Lamant L, Vigarios E, Mazieres J, Delord J. Dermatologic complications of anti-PD-1/PD-L1 immune checkpoint antibodies. Curr Opin Oncol. 2016;28:254-63.

13. Hassel J, Heinzerling L, Aberle J, Bähr O, Eigentler T, Grimm MO, et al. Combined immune checkpoint blockade (anti-PD-1/anti-CTLA-4): Evaluation and management of adverse drug reactions. Cancer Treat Rev. 2017;57:36-49.

14. Freeman-Keller M, Kim Y, Cronin H, Richards A, Gibney G, Weber J. Nivolumab in resected and unresectable metastatic melanoma: characteristics of immune-related adverse events and association with outco- 
mes. Clin Cancer Res. 2016;22:886-94.

15. Sanlorenzo M, Vujic I, Daud A, Algazi A, Gubens M, Luna S, et al. Pembrolizumab cutaneous adverse events and their association with disease progression. JAMA Dermatol. 2015;151:1206-12.

16. Khoja L, Day D, Wei-Wu Chen T, Siu LL, Hansen AR. Tumour- and class-specific patterns of immune-related adverse events of immune checkpoint inhibitors: a systematic review. Ann Oncol. 2017;28:237785.

17. Belum V, Benhuri B, Postow M, Hellmann M, Lesokhin A, Segal N, et al. Characterisation and management of dermatologic adverse events to agents targeting the PD-1 receptor. Eur J Cancer. 2016;60:12-25.

18. Hofmann L, Forschner A, Loquai C, Goldinger S, Zimmer L, Ugurel S, et al. Cutaneous, gastrointestinal, hepatic, endocrine, and renal side-effects of anti-PD-1 therapy. Eur J Cancer. 2016;60:190-209.

19. Minkis K, Garden B, Wu S, Pulitzer M, Lacouture M. The risk of rash associated with ipilimumab in patients with cancer: a systematic review of the literature and meta-analysis. J Am Acad Dermatol. 2013;69:e121-8.

20. Curry JL, Tetzlaff MT, Nagarajan P, Drucker C, Diab A, Hymes SR, et al. Diverse types of dermatologic toxicities from immune checkpoint blockade therapy. J Cutan Pathol. 2017;44:158-76.

21. Naidoo J, Page D, Li B, Connell L, Schindler K, Lacouture M, et al. Toxicities of the anti-PD-1 and anti-PD-L1 immune checkpoint antibodies. Ann Oncol. 2015;26:2375-91.

22. Joseph R, Cappel M, Goedjen B, Gordon M, Kirsch B, Gilstrap C, et al. Lichenoid dermatitis in three patients with metastatic melanoma treated with anti-PD-1 therapy. Cancer Immunol Res. 2015;3:18-22.

23. Tetzlaff M, Nagarajan $P$, Chon S, Huen A, Diab A, Omar P, et al. Lichenoid dermatologic toxicity from immune checkpoint blockade therapy: a detailed examination of the clinicopathologic features. Am J Dermatopathol. 2017;39:121-9.

24. Hwang S, Carlos G, Wakade D, Byth K, Kong B, Chou S, et al. Cutaneous adverse events (AEs) of anti-programmed cell death (PD)-1 therapy in patients with metastatic melanoma: a single-institution cohort. $J$ Am Acad Dermatol. 2016;74:455-61.

25. Shi V, Rodic N, Gettinger S, Leventhal J, Neckman J, Girardi M, et al. Clinical and histologic features of lichenoid mucocutaneous eruptions due to anti-programmed cell death 1 and anti-programmed cell death ligand 1 immunotherapy. JAMA Dermatol. 2016;152:1128-36.

26. Collins L, Chapman M, Carter J, Samie F. Cutaneous adverse effects of the immune checkpoint inhibitors. Curr Probl Cancer. 2017;41:125-8.

27. Matsumura N, Ohtsuka M, Kikuchi N, Yamamoto T. Exacerbation of psoriasis during nivolumab therapy for metastatic melanoma. Acta Derm Venereol. 2016;96:259-60.

28. Bonigen J, Raynaud-Donzel C, Hureaux J, Kramkimel N, Blom A, Jeudy G, et al. Anti-PD1-induced psoriasis: a study of 21 patients. J Eur Acad Dermatol Venereol. 2017;31:e254-7.

29. Dulos J, Carven G, van Boxtel S, Evers S, Driessen-Engels L, Hobo W, et al. PD-1 blockade augments Th1 and Th17 and suppresses Th2 responses in peripheral blood from patients with prostate and advanced melanoma cancer. J Immunother. 2012;35:169-78.

30. Lacouture M, Wolchok J, Yosipovitch G, Kähler K, Busam K, Hauschild A. Ipilimumab in patients with cancer and the management of dermatologic adverse events. J Am Acad Dermatol. 2014;71:161-9.

31. Goldinger S, Stieger P, Meier B, Micaletto S, Contassot E, French L, et al. Cytotoxic cutaneous adverse drug reactions during Anti-PD-1 therapy. Clin Cancer Res. 2016;22:4023-9.

32. Saw S, Lee H, Ng Q. Pembrolizumab-induced Stevens-Johnson syndrome in non-melanoma patients. Eur J Cancer. 2017;81:237-9.

33. Dasanu C. Late-onset Stevens-Johnson syndrome due to nivolumab use for hepatocellular carcinoma. J Oncol Pharm Pract. 2019;1078155219830166.

34. Vivar K, Deschaine M, Messina J, Divine J, Rabionet A, Patel N, et al. Epidermal programmed cell death-ligand 1 expression in TEN associated with nivolumab therapy. J Cutan Pathol. 2017;44:381-4.

35. Mirza S, Hill E, Ludlow S, Nanjappa S. Checkpoint inhibitor-associated drug reaction with eosinophilia and systemic symptom syndrome. Melanoma Res. 2017;27:271-3.

36. Hwang S, Carlos G, Wakade D, Sharma R, Fernandez-Penas P. Ipilimumab-induced acute generalized exanthematous pustulosis in a patient with metastatic melanoma. Melanoma Res. 2016;26:417-20.

37. Page B, Borradori L, Beltraminelli H, Yawalkar N, Hunger R. Acute generalized exanthematous pustulosis associated with ipilimumab and nivolumab. J Eur Acad Dermatol Venereol. 2018;32:e256-7.

38. Weber JS, Kähler KC, Hauschild A. Management of immune-related adverse events and kinetics of response with ipilimumab. J Clin Oncol. 2012;30:2691-7.
39. Postow MA. Managing immune checkpoint-blocking antibody side effects. Am Soc Clin Oncol Educ Book. 2015;76-83.

40. Antonia SJ, Villegas A, Daniel D, Vicente D, Murakami S, Hui R, et al. Durvalumab after chemoradiotherapy in stage III non-small-cell lung cancer. N Engl J Med. 2017;377:1919-29.

41. Donaldson M, Owen JL, Chae YK, Choi JN. Management of persistent pruritus and lichenoid reaction secondary to nivolumab with narrowband ultraviolet B phototherapy. Front Oncol. 2018;8:405

42. Naidoo J, Schindler K, Querfeld C, Busam K, Cunningham J, Page DB et al. Autoimmune bullous skin disorders with ilmmune checkpoint inhibitors targeting PD-1 and PD-L1. Cancer Immunol Res. 2016;4:383-9.

43. Rodríguez-Lomba E, Molina-López I, Suárez-Fernández R, Baniandrés-Rodríguez O. Vitiligo-like lesions and immune checkpoint inhibition therapy: is it truly an adverse event exclusive to patients with melanoma? Clin Exp Dermatol. 2018;43:598-9.

44. Kosche $\mathrm{C}$, Mohindra $\mathrm{N}$, Choi J. Vitiligo in a patient undergoing nivolumab treatment for non-small cell lung cancer. JAAD Case Rep. 2018;4:10424.

45. Liu RC, Consuegra G, Chou S, Fernandez Peñas P. Vitiligo-like depig mentation in oncology patients treated with immunotherapies for nonmelanoma metastatic cancers. Clin Exp Dermatol. 2019;44:643-6.

46. Hua C, Boussemart L, Mateus C, Routier E, Boutros C, Cazenave H, et al. Association of vitiligo with tumor response in patients with metastatic melanoma treated with pembrolizumab. JAMA Dermatol. 2016;152:4551.

47. Nakamura Y, Tanaka R, Asami Y, Teramoto Y, Imamura T, Sato S, et al. Correlation between vitiligo occurrence and clinical benefit in advanced melanoma patients treated with nivolumab: a multi-institutional retrospective study. J Dermatol. 2017;44:117-22.

48. Larsabal M, Marti A, Jacquemin C, Rambert J, Thiolat D, Dousset L, et al. Vitiligo-like lesions occurring in patients receiving anti-programmed cell death-1 therapies are clinically and biologically distinct from vitiligo. J Am Acad Dermatol. 2017;76:863-70.

49. Boniface K, Dutriaux C, Prey S, Taieb A, Seneschal J. Vitiligo-like lesions in patients receiving anti-programmed cell death-1 therapies are distinct from spontaneously occurring active vitiligo. J Am Acad Dermatol. 2018;78:e17-8.

50. Carlos G, Anforth R, Chou S, Clements A, Fernandez-Peñas P. A case of bullous pemphigoid in a patient with metastatic melanoma treated with pembrolizumab. Melanoma Res. 2015;25:265-8.

51. Tanita K, Fujimura T, Kambayashi Y, Tsukada A, Sato Y, Hashimoto A et al. Intensity-modulated radiotherapy triggers onset of bullous pemphigoid in a patient with advanced melanoma treated with nivolumab. Case Rep Oncol. 2018;11:114-8.

52. Hanley T, Papa S, Saha M. Bullous pemphigoid associated with ipilimumab therapy for advanced metastatic melanoma. JRSM Open. 2018;9:2054270418793029.

53. Sheik Ali S, Goddard A, Luke J, Donahue H, Todd D, Werchniak A, et al. Drug-associated dermatomyositis following ipilimumab therapy: a novel immune-mediated adverse event associated with cytotoxic T-lymphocyte antigen 4 blockade. JAMA Dermatol. 2015;151:195-9.

54. Liewluck T, Kao J, Mauermann M. PD-1 Inhibitor-associated myopathies: emerging immune-mediated myopathies. J Immunother. 2018;41:20811.

55. Shao K, McGettigan S, Elenitsas R, Chu EY. Lupus-like cutaneous reaction following pembrolizumab: an immune-related adverse event associated with anti-PD-1 therapy. J Cutan Pathol. 2018;45:74-7.

56. Zitouni N, Arnault JP, Dadban A, Attencourt C, Lok C, Chaby G. Subacute cutaneous lupus erythematosus induced by nivolumab: two case reports and a literature review. Melanoma Res. 2019;29:212-5.

57. Jin HT, Ahmed R, Okazaki T. Role of PD-1 in regulating T-cell immunity. Curr Top Microbiol Immunol. 2011;350:17-37.

58. Le Burel S, Champiat S, Routier E, Aspeslagh S, Albiges L, Szwebe TA, et al. Onset of connective tissue disease following anti-PD1/PD-L1 cancer immunotherapy. Ann Rheum Dis. 2018;77:468-70.

59. Cappelli L, Gutierrez A, Baer A, Albayda J, Manno R, Haque U, et al. Inflammatory arthritis and sicca syndrome induced by nivolumab and ipilimumab. Ann Rheum Dis. 2017;76:43-50.

60. Tocut M, Brenner R, Zandman-Goddard G. Autoimmune phenomena and disease in cancer patients treated with immune checkpoint inhibitors. Autoimmun Rev. 2018;17:610-6.

61. Zamani M, Aslani S, Salmaninejad A, Javan M, Rezaei N. PD-1/PD-L and autoimmunity: a growing relationship. Cell Immunol. 2016;310:2741.

62. Daxini A, Cronin K, Sreih A. Vasculitis associated with immune checkpoint inhibitors-a systematic review. Clin Rheumatol. 2018;37:2579-84. 
63. Munoz J, Guillot B, Girard C, Dereure O, Du-Thanh A. First report of ipilimumab-induced Grover disease. Br J Dermatol. 2014;171:1236-7.

64. Koelzer VH, Buser T, Willi N, Rothschild SI, Wicki A, Schiller P, et al. Grover's-like drug eruption in a patient with metastatic melanoma under ipilimumab therapy. J Immunother Cancer. 2016;4:47.

65. Chen WS, Tetzlaff MT, Diwan H, Jahan-Tigh R, Diab A, Nelson K, et al. Suprabasal acantholytic dermatologic toxicities associated checkpoint inhibitor therapy: a spectrum of immune reactions from paraneoplastic pemphigus-like to Grover-like lesions. J Cutan Pathol. 2018;45:764-73.

66. Yatim N, Mateus C, Charles P. Sarcoidosis post-anti-PD-1 therapy, mimicking relapse of metastatic melanoma in a patient undergoing complete remission. Rev Med Interne. 2018;39:130-3.

67. Cotliar J, Querfeld C, Boswell WJ, Raja N, Raz D, Chen R. Pembrolizumab-associated sarcoidosis. JAAD Case Rep. 2016;2:290-3.

68. Kim C, Gao J, Shannon VR, Siefker-Radtke A. Systemic sarcoidosis first manifesting in a tattoo in the setting of immune checkpoint inhibition. BMJ Case Rep. 2016;2016.

69. Danlos FX, Pagès C, Baroudjian B, Vercellino L, Battistella M, Mimoun $M$, et al. Nivolumab-induced sarcoid-like granulomatous reaction in a patient with advanced melanoma. Chest. 2016;149:e133-6.
70. Abdel-Wahab N, Shah M, Suarez-Almazor ME. Adverse events Associated with immune checkpoint blockade in patients with cancer: a systematic review of case reports. PLoS One. 2016;11:e0160221.

71. Gormley R, Wanat K, Elenitsas R, Giles J, McGettigan S, Schuchter L, et al. Ipilimumab-associated Sweet syndrome in a melanoma patient. J Am Acad Dermatol. 2014;71:e211-3.

72. Rudolph B, Staib F, Von Stebut E, Hainz M, Grabbe S, Loquai C. Neutrophilic disease of the skin and intestines after ipilimumab treatment for malignant melanoma - simultaneous occurrence of pyoderma gangrenosum and colitis. Eur J Dermatol. 2014;24:268-9.

73. Kinoshita-Ise M, Foster F, Shear N. Immune checkpoint inhibitor-related alopecia: insight into the pathophysiology utilizing non-invasive diagnostic techniques. J Dermatol. 2019;46:e152-3.

74. Rivera N, Boada A, Bielsa MI, Fernández-Figueras MT, Carcereny E, Moran MT, et al. Hair repigmentation during immunotherapy treatment with an anti-programmed cell death 1 and anti-programmed cell death ligand 1 agent for lung cancer. JAMA Dermatol. 2017;153:1162-5.

75. Vigarios E, Epstein JB, Sibaud V. Oral mucosal changes induced by anticancer targeted therapies and immune checkpoint inhibitors. Support Care Cancer. 2017;25:1713-39. 